\title{
A Study on Image Segmentation Techniques
}

\author{
S. Abdul Saleem ${ }^{1}$ and G. Vinitha ${ }^{2}$ \\ ${ }^{1}$ Associate Professor, ${ }^{2}$ Research Scholar \\ ${ }^{1 \& 2}$ Department of Computer Science, Jamal Mohamed College, Tiruchirappalli, Tamil Nadu, India \\ E-Mail: vinithavini452@gmail.com
}

\begin{abstract}
Image processing is a technique to transform an image into digital form and implement some operations on it; in order to acquire an improved image or to abstract some useful information from it. It is a kind of signal exemption in which input is image, like video frame or photograph and output may be image or characteristics related with that image. Segmentation partitions an image into separate regions comprising each pixel with similar attributes. To be significant and useful for image analysis and clarification, the regions should powerfully relate to depicted objects or features of interest. Meaningful segmentation is the first step from lowlevel image processing converting a grey scale or color image into one or more other images to high-level image depiction in terms of objects, features, and scenes. The achievement of image analysis depends on reliability of segmentation, but an exact partitioning of an image is mostly a very challenging problem.
\end{abstract}

Keywords: Image Processing, Segmentation, Image, Segmentation Classification

\section{INTRODUCTION}

Due to the initiation of computer technology imageprocessing techniques have become gradually significant in a wide range of applications. Image segmentation is a definitive subject in the field of image processing and also is a hotspot and attention of image processing techniques. Numerous general-purpose algorithms and techniques have been established for image segmentation [1]. Meanwhile there is no common solution to the image segmentation problem; these techniques often have to be joined with domain knowledge in order to efficiently resolve an image segmentation problem for problem domain. Image segmentation is a vital process in many image, video, and computer vision applications. It is often used to divide an image into distinct regions, which perfectly correspond to diverse real-world objects [2]. It is a serious step towards content study and image understanding.

In everyday life, new technologies are developing in the field of Image processing; particularly in the domain of segmentation. Segmentation is the utmost vital part in image processing. Fence off a complete image into numerous parts which is something more important and easier for additional process. These numerous parts that are re-joined will refuge the complete image. Segmentation may also depend on many features that are confined in the image. It may be any color or texture [3]. Before demonizing an image, it is segmented to improve the original image. The key motto of segmentation is to decrease the information for easy analysis. Segmentation is also beneficial in Image Analysis and Image Compression.

Primary Object Segmentation (POS) is the job to section a single key object from the background in a video arrangement, where the key object means the utmost often appearing and relevant object in the video. POS is related to several vision tasks, comprising video summarization, action recognition, and object class learning. However, POS is inspiring due to several difficulties such as object deformation, occlusion, and background clutters[4]. Particularly, without user annotations or any previous information about a key object (e.g. its class), it is difficult to isolate the key object from the background. POS is strictly associated to video object segmentation (VOS).

\section{IMAGE PROCESSING}

Image processing mainly comprises the following three steps [5]:

1. Introducing the image with optical scanner or via digital photography.

2. Analyzing and deploying the image which contains data compression, image improvement and spotting patterns that are not to human eyes like satellite photographs.

3. Output is the final stage in which outcome can be change image or report which is based on image analysis.

The purpose of image processing is separated into 5 groups. They are:

a. Visualization: Detect the objects that are not visible.

b. Image Sharpening and Restoration- To construct a better image.

c. Image Retrieval: Pursue for the image of interest.

d. Measurement of Pattern: Measures numerous objects in an image.

e. Image Recognition: Differentiate the objects in an image.

\section{III.SEGMENTATION}

In computer vision, image segmentation is the practice of subdividing a digital image into numerous segments (sets of pixels, also identified as super-pixels). The aim of segmentation is to shorten and/or modify the representation of an image into something that is more important and 
easier to analyses. Image segmentation is usually used to trace objects and boundaries (lines, curves etc.) in images. More specifically, image segmentation is the practice of allocating a label to every pixel in an image such that pixels with the same label share assured characteristics.

The outcome of image segmentation is a set of segments that jointly cover the complete image, or a set of contours mined from the image. Every pixel in a region is related with respect to some characteristic or computed property, such as intensity, color, or texture. Nearby regions are meaningfully dissimilar with respect to the same characteristics. Once applied to a stack of images (usual in medical imaging), resultant contours after image segmentation can be used to build 3D reconstructions with the help of interruption algorithms like Marching cubes. Segmentation algorithms usually are based on one of two basis properties of intensity values [6].

1. Discontinuity: To partition an image based on rapid changes in intensity (such as edges).

2. Similarity: To partition an image into regions those are related rendering to a set of predefined criteria.

\section{IV.SEGMENTATION CLASSIFICATION AND ITS METHODS}

Image segmentation techniques or methods are categorized into two main categories[1]. They are:

1. Layer-Based Segmentation Methods

2. Block-Based Segmentation Methods.

1. Layer-Based Segmentation Methods: Layered model for object discovery and image segmentation that composites the output of a bank of object detectors in order to describe shape masks and clarify the appearance, depth ordering, and that estimates both class and instance segmentation.

2. Block-Based Segmentation Method: This is based on numerous features found in the image. The feature focused is color information that is used to make histograms, or information about the pixels that specify edges or boundaries or texture information.

Block-Based Image Segmentation methods are characterized on two properties: discontinuity and similarity into two groups:

a. Region Based Methods: created on discontinuities.

b. Edge or Boundary Based Methods: created on similarity.

a. Region Based Methods: Split the complete image into sub regions or clusters, e.g. every pixel with same grey level in one region.

i. Clustering: K-mean: separate an image into $\mathrm{K}$ groups or groups by adding points (p); to the cluster where the variance between the point and the mean is smallest. ii. Split and Merge: Two parts: Firstly the complete image which is taken as a solo region is continually divided until no more separations are possible. Quad tree is a piercing data structure. Two regions are combined if they are adjacent and similar. Unification is frequent until no more integration is possible.

iii. Normalized Cuts: Normalized cuts target at optimal splitting by decreasing number of regions. This method is based on graph theory.

iv. Region Growing: Region growing is one of widespread methods. Starts with a pixel and will go on adding the pixels based on comparison to the region. Repeated till all pixels belong to some region.

v. Threshold: Threshold segmentation techniques clustered in classes:

a. Local techniques are based on the confined properties of the pixels and their neighborhoods.

b. Global techniques segments an image on the basis of information attain globally (e.g. by using image histogram; global texture properties).

c. Split, merge and growing techniques use both the ideas of homogeneity and geometrical proximity in order to attain good segmentation outcomes.

b. Edge or Boundary Based Methods: Edges are identified to detect the breaks in the image. Edges on the region are outlined by finding the pixel value and it is related with the neighboring pixels. For this classification they use together fixed and adaptive feature of Support Vector Machine.

\section{i. Edge Detection Methods:}

a. Roberts Detection: The Roberts operator accomplishes a simple, rapid to compute, 2-D spatial gradient measurement on an image. Its high-pointed regions of great spatial gradient which frequently correspond to edges.

b. Prewitt Detection: It is a discrete differentiation operator; computing an estimate of the gradient of the image greatness function. At every point in the image, the outcome of the Prewitt operator is either the corresponding gradient vector or the norm of this vector.

c. Sobel Detection: Sobel detection is used in image processing; mainly within edge detection algorithms where it makes an image emphasizing edges.

\section{ii. Edge Detection Soft Computer approaches:}

a. Fuzzy Logic Based Approach: Pixels are separated into fuzzy sets i.e. every pixel may belong partially to numerous sets and regions of image.

b. Genetic Algorithm Approach: Develops from the evolution theory comprises of three major operations: selection, crossover, and mutation. GA used in pattern's recognition applications. 
c. Neural Network Approach: The network "learns" by altering the interconnection, weights, between layers, and simplifies related output for a set of input data.

\section{RELATED WORKS}

Image segmentation is an essential process in many image and video processing applications. In this literature survey, we study and analyze the various segmentation techniques. The Table I shows the comparative analysis of some relevant segmentation techniques.

K. Zhang et al., [7] done semantic segmentation in weak supervision through classifier valuation where the classifier parameters are firstly sampled at random and then the super pixel classifiers are calculated by measuring the distance among the ground-truth negative samples and the expected positive samples. It should be pointed out that all above methods are based on the assumption that the specified image-level labels for training are accurate and complete; which is not practical in numerous real-world applications. It is a genuine problem where the end objective is pixellevel labels but the input is noisy image-level annotations.

Shottonet al., [8] applied semantic segmentation by including shape-texture, color, location and edge clues in a CRF model over image pixels. This model is protracted in the follow-up works

C. Rotheret al., [9] introduced Co-segmentation which uses histogram matching; to concurrently segment the common object in a duo of images. This paper has motivated much follow-up work, including co-segmentation methods based on maximum flow optimization to increase efficiency, intelligent scribble guidance to facilitate processing of bigger image sets; discriminative clustering that handles better variation in foreground appearance and rank constraints for scale invariance.

H. Fu et al., [10] excerpts common candidates from numerous videos by using an object based co-selection graph, which is expressed as a classical CRF energy minimization problem. However, these techniques have a significant assumption that the common foreground objects must appear in all the images/videos in the set. This strong assumption significantly limits the application field of these methods. If the common foreground object happens to be missing in one of the images; though these methods will segment an unrelated region.

Wei Zhang et al., [11] propose a semantic segmentation algorithm that is accomplished from image-level labels instead of pixel-level labels and can handle noisy labels. They take benefit of a unified conditional random field to include numerous contextual relations such as the relations between semantic concepts, spatial neighborhood clues, visual appearance, label correlations, and label consistency among image-level and pixel-level. Visual features are mined by deep convolution neural network and latent semantic concept distribution. Label correlations are wellread by instantaneously exploiting how often two labels cooccur in the same image and what pairs of labels commonly overlap. Investigational results on two real-world image datasets PASCAL VOC2007 and SIFT-Flow prove that the proposed approach beats most of the current methods and attains a promising performance in noisy condition as well.

Santiago Aja-Fern'andez et al., [12] proposal is the use of the spatial collection step, which makes our technique able to overwhelmed noise-related problems that traditional ways cannot cope with. Their first effort to define common purpose thresholding aggregations have shown worthy results, and can possibly get even improved when applied with constraints appropriate for specific segmentation goals. The methodology planned has the following benefits: (1) It is totally instinctive, and does not need human intervention; (2) the hard thresholding decision is suspended to the final stage; so all the spatial operations are completed before taking into account the different memberships; (3) spatial aggregations create the thresholding more robust to noise and artifacts; (4) it can benefit from the use of exact thresholding techniques in the first step; and (5) the spatial aggregations can be specifically planned for a specific application, and heuristic information can be added through a fuzzy inference rule base.

Huazhu Fu et al., [13] proposed an object-based cosegmentation technique for RGBD images that creates active use of depth information. With a fully associated graph structure and mutex constraints, their technique is able to correctly deal with image sets that comprise noisy images with more than or less than one communal foreground object.

Hai Min et al., [14] The Intensity-Texture model, which includes intensity term into texture term, was proposed to segment difficult two-phase nature images. On one hand, the intensity term based on global division algorithm has been built and has more benefits than CV model in catching intensity information. The proposed ASLVD algorithm can abstract the amplitude and frequency components of local intensity variation, which is used to reflect the texture feature efficiently. Lastly, the intensity term and the texture term are balancing and combined into level set method to build Intensity-Texture model. The experiments have testified the efficiency of the Intensity-Texture model for segmenting complex two-phase nature images. The proposed Intensity-Texture model is mostly used to segment complex two-phase images.

IzharHaq et al., [15] proposed and proved a fuzzy logic based edge detection algorithm for smooth and noisy images. The established method employs a $3 \times 3$ mask directed by fuzzy rule set for edge detection in noisy images. The advanced algorithm is also related with other conventional and formerly developed fuzzy logic based edge detection techniques. It is understandable from the experimental outcomes that in case of smooth and noisy images the developed system provides improved results. 
TABLE I COMPARATIVE STUDY

\begin{tabular}{|c|c|c|c|c|}
\hline Authors & Algorithm / Model & Dataset & Process & Accuracy in Result \\
\hline K. Zhang et al., [7] & $\begin{array}{l}\text { Iterative Merging Update } \\
\text { (IMU) algorithm }\end{array}$ & Real World Noisy Images & Segmentation & $\begin{array}{l}\text { Trained - 87\% } \\
\text { Tested - 69\% } \\
\end{array}$ \\
\hline Shotton et al.,[8] & CRF model & Noisy Images & Segmentation & $72.2 \%$ \\
\hline C. Rother et al., [9] & $\begin{array}{l}\text { A generative model for co- } \\
\text { segmentation }\end{array}$ & Input pair images & Segmentation & $42 \%$ to $58 \%$ \\
\hline H. Fu et al., [10] & $\begin{array}{l}\text { Multi-State Selection Graph } \\
\text { Model (MSG) }\end{array}$ & Video Clips & Segmentation & $\begin{array}{l}\text { Better Accuracy than } \\
\text { Existing }\end{array}$ \\
\hline Wei Zhang et al., [11] & $\begin{array}{l}\text { Semantic Segmentation } \\
\text { Algorithm }\end{array}$ & $\begin{array}{l}\text { SIFT-Flow Dataset - Noisy } \\
\text { Image }\end{array}$ & Segmentation & $\begin{array}{l}10 \% \text { of noise }-32.8 \% \\
25 \% \text { of noise }-32.4 \% \\
50 \% \text { of noise }-29.8 \% \\
75 \% \text { of noise }-22.3 \%\end{array}$ \\
\hline $\begin{array}{l}\text { Santiago Aja- } \\
\text { Fern'andez et al., [12] }\end{array}$ & $\begin{array}{l}\text { Spatial-Based Thresholding } \\
\text { Methodology }\end{array}$ & $\begin{array}{l}\text { Berkeley Segmentation Dataset } \\
\text { And Benchmark (BSDS300) }\end{array}$ & Segmentation & $\begin{array}{l}\text { Accurate than existing } \\
\text { approach }\end{array}$ \\
\hline Huazhu Fu et al., [13] & $\begin{array}{l}\text { Object-Based Co-Segmentation } \\
\text { Technique }\end{array}$ & $\begin{array}{l}\text { RGBD Co-Segmentation } \\
\text { Dataset - Noisy Image }\end{array}$ & Segmentation & $92.3 \%$ \\
\hline Hai Min et al.,[14] & $\begin{array}{l}\text { Intensity-Texture Model } \\
\text { ASLVD Algorithm }\end{array}$ & Nature Images - Noisy Image & Segmentation & More accurate \\
\hline IzharHaqet al.,[15] & $\begin{array}{l}\text { Fuzzy Logic Based Edge } \\
\text { Detection Algorithm }\end{array}$ & Noisy image & Segmentation & $\begin{array}{l}\text { Sensitivity }-89 \% \\
\text { Specificity }-96 \%\end{array}$ \\
\hline
\end{tabular}

\section{CONCLUSION}

It is among quickly developing technologies today, with its applications in numerous aspects of a business. Image Processing forms essential research area within engineering and computer science too. Imagesegmentation is the method of separating an image into several parts. This is usually used to find objects or other related information in digital images. There are several different ways to achieve image segmentation. Image segmentation is a challenge in image processing and the researchers would calculate their image segmentation techniques by using one or more of the valuation methods. Segmentation can be applied on any variety of images. This paper summarizes various segmentation techniques and their performance.

\section{REFERENCES}

[1] Nida M. Zaitoun, and Musbah J. Aqel, "Survey on Image Segmentation Techniques", International Conference on Communication, Management and Information Technology (ICCMIT), pp. 797-806, 2015.

[2] N.Senthil kumaran, and S.Vaithegi, "Image Segmentation by using Thresholding Techniques for Medical Images", Computer Science \& Engineering: An International Journal, Vol. 6, Feb. 2016.

[3] R. Yogamangalam, and B.Karthikeyan, "Segmentation Techniques Comparison in Image Processing", International Journal of Engineering and Technology (IJET), Vol. 5, Mar. 2013.

[4] Yeong Jun Koh, and Chang-Su Kim, "Primary Object Segmentation in Videos Based on Region Augmentation and Reduction”,30th IEEE Conference on Computer Vision and Pattern Recognition., pp.74177425, 2017.
[5] Rose Mary [Online] Available at: https://www.engineersgarage.com/ articles/image-processing-tutorial-applications

[6] [Online] Available at: https://en.wikipedia.org/wiki/Image_segmentat ion,2019

[7] K. Zhang, W. Zhang, Y. Zheng, and X. Xue,"Sparse reconstruction for weakly supervised semantic segmentation”,IJCAI, pp. 1889-1895, 2013.

[8] J. Shotton, J. Winn, C. Rother, and A. Criminisi,“Textonboost: Joint appearance, shape and context modeling for multi-class object recognition and segmentation", European Conference on Computer Vision (ECCV), pp.1-15,2006.

[9] C. Rother, T. Minka, A. Blake, and V. Kolmogorov. "Cosegmentation of Image Pairs by Histogram Matching Incorporating a Global Constraint into MRFs”, CVPR,Vol.1, 2006

[10] H. Fu, D. Xu, B. Zhang, and S. Lin, “Object-based multiple foreground video co-segmentation”, Conference on Computer Vision and Pattern Recognition (CVPR),pp. 3166-3173, 2014.

[11] Wei Zhang, Sheng Zeng, Dequan Wang, and XiangyangXue,’WeaklySupervisedSemanticSegmentationforSocialI mages", Conference on Computer Vision and Pattern Recognition (CVPR), pp. 2718-2726,2015.

[12] Santiago Aja-Fern'andez, Ariel Hern'an Curiale, and Gonzalo VegasS'anchez-Ferrero,"A local fuzzy thresholding methodology for multiregion image segmentation”, Knowledge Based Systems, Jan. 2015.

[13] Huazhu Fu Dong Xu Stephen Lin Jiang Liu, "Object-based RGBD Image Co-segmentation with Mutex Constraint”, in IEEE Conference on Computer Vision and Pattern Recognition (CVPR), pp. 44284436, 2015.

[14] Hai Min, Wei Jia, Xiao-Feng Wang, Yang Zhao, Rong-Xiang Hu, Yue-Tong Luo, Feng Xue, and Jing-Ting Lu,“An Intensity-Texture model based level set method for image segmentation", Pattern Recognition, pp.1547-1562, 2015.

[15] Izhar Haq, Shahzad Anwar, Kamran Shah , Muhammad Tahir Khan and Shaukat Ali Shah, "Fuzzy Logic Based Edge Detection in Smooth and Noisy Clinical Images”, PLOS ONE, Sep. 2015. 